\title{
Transparency in Reimbursement Decisions: In Whose Best Interest?
}

\author{
Tania Stafinski · Devidas Menon
}

Published online: 28 May 2014

(C) Springer International Publishing Switzerland 2014

According to the Oxford English Dictionary, 'transparency' is defined as "Easily seen through or understood; easily discerned; obvious; (of a person, statement, etc.) frank, open, ingenuous" [1]. In the context of decisionmaking, 'transparency' comprises a key condition of procedural justice. Procedural justice concerns the fairness of processes by which decisions are made [2]. For the past decade, 'procedural justice' has, implicitly or explicitly, served as a guiding principle for many healthcare decision-making bodies, particularly those tasked with determining which services to provide and under what conditions. This has, in part, been attributed to increased awareness of recent scholarly frameworks for making healthcare funding decisions (e.g., accountability for reasonableness [3]). These frameworks embrace Rawlsian notions of 'pure procedural justice,' which state that there is no criterion for what constitutes a 'just' outcome other than the procedure itself [2]. They are built upon the premise that we, as a society, are unlikely to fully agree on coverage or reimbursement decisions, especially when the outcomes of those decisions mean access to a service/ therapy for some and denial of a service/therapy to others. However, we may be able to agree on the process used to arrive at the outcomes (i.e., how decisions are made), and if we accept the process, we are more likely to accept the outcomes. It is not surprising that this argument has resonated with centralized drug reimbursement review (CDR) organizations, where individuals involved represent a number of stakeholder communities and competing interests and there is no shared objective. Those communities include industry, healthcare providers/clinicians, patients,

T. Stafinski $(\bowtie) \cdot$ D. Menon

University of Alberta, Edmonton, AB, Canada

e-mail: tanias@ualberta.ca and payers. In the absence of a shared objective, claims of procedural justice and, therefore, transparency, become all the more important. For governments, transparency is a means of demonstrating accountability to the public. For patients, it helps to ensure that their perspectives matter and have been considered during decision-making processes. For industry, it increases their understanding of what healthcare systems will pay in return for certain health benefits, informing investment decisions around the development of new technologies.

The past 10 years have seen a plethora of academic papers discussing and debating the transparency of CDR processes around the world. Most have assessed 'transparency' against (1) factors related to the availability of information about the process on a publicly accessible website (including the health technology assessment used to inform review committee deliberations, decision criteria, outcomes of the deliberations, and rationale) or (2) the extent to which deliberations within review committees are open to different stakeholder communities and/or the general public [4, 5]. Within papers taking the first approach, there is often little, if any, discussion of whether these are appropriate measures of 'transparency.' Can 'transparency' be legitimately defined or assessed on the basis of the availability of information without opportunities to verify the credibility of that information? Is a 'trust me' approach to how decisions are made acceptable? Given that criticisms from stakeholder communities have stayed somewhat the same, despite the evolution of these processes over the years, it would appear that the answer is "no." Calls from industry, patients, and providers for greater 'transparency' in reimbursement decisions remain, and surge when an announcement of a negative decision is made, as they have since the inception of CDR processes. The 'black box' still exists. 
Few would argue that the critical step in any CDR process is formulation of the decision (or recommendation, depending on the jurisdiction). For that reason, we've chosen to focus on this point. If we take a narrow view of 'transparency' and assume it may be operationalized simply through an open invitation to attend CDR deliberations, why hasn't this happened? Why do so few review bodies hold meetings even partially in public? There are likely many reasons, but we would argue that they predominantly relate to unresolved issues with and concerns over how information emerging from such meetings is used. Results of research into the perspectives of individuals engaged in priority-setting and coverage decision-making around different health services, including drugs, have highlighted fears of being 'demonized,' distrust and tension among the different stakeholder communities, and 'blame avoidance' as explanations for practicing a 'behind closed-doors' approach [6-8]. Decision-makers, on multiple occasions, have stated, "We don't wake up in the morning and ask ourselves, 'Who can we deny a drug to today'?" Each is all too aware of colleagues who became the casualties of what are commonly politically polarizing decisions. Let us not forget that the stakes surrounding these decisions can be high and it is a zero sum game. For industry, especially small or single product companies, a negative decision can derail years of dedicated, focused research and development efforts, destabilize business, and weaken the confidence of shareholders. For patients and their loved ones, particularly those for whom there are no other active treatment options, a negative decision can be painful and heartbreaking, dashing hopes for some health benefit, even if it is small. Legitimately frustrated and often desperate, they resort to public pressure, which has, unquestionably, been proven to be a powerful tool. In recent years, we have witnessed the impressive force of social media, with the launch of Facebook and Twitter campaigns seeking coverage for a therapy. They remind us of both the admirable and not-so-admirable aspects of society. Regrettably, 'posts' and 'tweets' target individual decision-makers, blaming, vilifying, and, in some cases, wishing ill-harm upon them. Thus, there is little wonder why decisionmakers and CDR processes have opted for a safer, less transparent approach to coverage deliberations. However, as we mentioned above, this assumes that 'transparency' in reimbursement decisions is defined by the process, which rests entirely with CDR bodies. We would argue that their role, while necessary, it is not sufficient. For 'procedural justice' to be realized, conditions of 'transparency' need to adopt a wider scope.

Drawing from inputs-processes-outputs (IPO) models of systems organization, 'transparency' may be more appropriately assessed according to three factors: (1) inputs, (2) processes, and (3) outputs [9]. 'Inputs' include all of the types and sources of information that flow into processes within an organization. 'Processes' provide the flow through which an organization achieves its purpose, and 'outputs' refer to what the organization "exports into the environment" (in our case, decisions). To achieve 'transparency,' conditions related to all three factors must be met. If we accept this position and apply it to reimbursement decisions, how would we fare? Regarding 'inputs,' most CDR bodies around the world use industry submissions as the primary source of information on the safety, efficacy, and cost-effectiveness (where required) of a drug [10]. Some also conduct or commission independent systematic reviews of the clinical evidence and/or economic evaluations to supplement industry submissions. However, they rely on data that are publicly available or provided by industry, the comprehensiveness of which is nearly impossible to confirm. Frequently, companies will not disclose full trial protocols, providing justifications related to commercial confidentiality [11]. Whether we feel these are compelling arguments for a drug well into its lifecycle is irrelevant. What matters is that CDR bodies, when faced with assessing the value of a drug, are seldom working with all that is known about it. Also, relationships among stakeholder communities who contribute supporting information are not always clear. Thus, before even considering the 'process' component, we've failed to meet transparency conditions, and in turn, increased the uncertainty under which decisions are made. CDR bodies, quite rightly, are risk-averse, and the greater the uncertainty, the greater the risk of making a 'wrong' decision. Regarding 'process,' while descriptions of procedures, decision criteria/factors, CDR committee governance, structure and membership, and sources of information considered may be public, deliberations, with a few exceptions, are not. Despite the availability of documents stating decisions and rationale, we know little about how different kinds and sources of evidence are interpreted and weighed against each other. Without that understanding, moving forward can be challenging. Regarding 'outputs,' in recent years, CDR bodies have made considerable efforts to ensure broad and timely dissemination of the outcomes of deliberations to stakeholder communities. Typically, summaries presenting a brief overview of the committee's deliberations and findings (i.e., recommendations or decisions) are prepared. Depending on the CDR body, companies may request removal of proprietary information, such as cost-effectiveness ratios, prior to their release. Unfortunately, such information is often key to making sense of the outcomes.

Thus, it seems that we still have some distance to go before optimal 'transparency' in reimbursement decisions is achieved. Getting there will first require consensus among CDR bodies and stakeholder communities that it is a shared responsibility. This, in itself, is a paradigm shift. 
Second, it will require a change in the current rules of engagement for reacting to information over which we may disagree. Neither of these ideas is particularly innovative. The fact that most CDR bodies have existed for well over a decade and we have yet to see either idea operationalized may serve as a sobering reality check. At the same time, 'transparency' in reimbursement decisions, as it stands now, does not appear to be in anybody's best interest.

Conflict of interest The authors declare that they have no conflicts of interest.

\section{References}

1. The new shorter Oxford English dictionary on historical principles. Oxford: Oxford University Press; 1993.

2. Rawls J. A theory of justice. Rev ed. Boston: Harvard University Press; 1999.

3. Daniels N, Sabin J. The ethics of accountability in managed care reform. Health Aff (Millwood). 1998;17(5):50-64.
4. Stafinski T, Menon D, Davis C, McCabe C. Role of centralized review processes for making reimbursement decisions on new health technologies in Europe. Clinicoecon Outcomes Res. 2011;3:117-86.

5. Menon D, Stafinski T. Role of patient and public participation in health technology assessment and coverage decisions. Expert Rev Pharmacoecon Outcomes Res. 2011;11(1):75-89.

6. Hood C. The blame game: spin, bureaucracy, and self-preservation in government. Princeton: Princeton University Press; 2011.

7. Should we make the priority setting process more explicit to the public? Priorities 2010: International Society on Priorities in Health Care (ISPHC) conference, Boston, 23-25 April 2010; Vancouver: International Society on Priorities in Health Care (ISPHC); 2010.

8. Vis B, Van Kersbergen K. Towards an open functional approach to welfare state change: pressures, ideas, and blame avoidance. Public Adm. 2013;91(4):840-54.

9. McGrath JE, Arrow H, Berdahl JL. The study of groups: past, present and future. Pers Soc Psychol Rev. 2000;4(1):95-105.

10. Stafinski T, Menon D, Philippon DJ, McCabe C. Health technology funding decision-making processes around the world: the same, yet different. Pharmacoeconomics. 2011;29(6):475-95.

11. Laupacis A. On bias and transparency in the development of influential recommendations. CMAJ. 2006;174(3):335-6. 